\title{
Les Chorèmes face à l'évolution des thématiques et pratiques de recherche, en agronomie et aménagement des territoires
}

\section{Sylvie Lardon et Marie Houdart}

\section{OpenEdition}

Journals

Édition électronique

URL : http://journals.openedition.org/mappemonde/574

DOI : 10.4000/mappemonde.574

ISSN : 1769-7298

Éditeur

UMR ESPACE

Référence électronique

Sylvie Lardon et Marie Houdart, « Les Chorèmes face à l'évolution des thématiques et pratiques de recherche, en agronomie et aménagement des territoires », Mappemonde [En ligne], 122 | 2017, mis en ligne le 08 décembre 2017, consulté le 20 juin 2019. URL : http://journals.openedition.org/ mappemonde/574 ; DOI : 10.4000/mappemonde.574

Ce document a été généré automatiquement le 20 juin 2019.

\section{c) (i) (2)}

La revue Mappemonde est mise à disposition selon les termes de la Licence Creative Commons Attribution - Pas d'Utilisation Commerciale - Partage dans les Mêmes Conditions 4.0 International. 


\title{
Les Chorèmes face à l'évolution des thématiques et pratiques de recherche, en agronomie et aménagement des territoires
}

\author{
Sylvie Lardon et Marie Houdart
}

\section{Agronomie et géographie, des synergies}

1 Voilà un peu plus de 25 ans paraissait le numéro de M@ppemonde intitulé « Gestion de l'espace rural, des pratiques aux modèles ", coordonné par Jean-Pierre Deffontaines, JeanPaul Cheylan et Sylvie Lardon (1990). Ayant pour origine une rencontre entre agronomes et géographes, l'objectif de ce numéro était de mettre à l'épreuve l'hypothèse de base qui fonde les chorèmes (Brunet, 1986): «Permettent-ils un regard nouveau sur les phénomènes étudiés? Où se situent-ils comme outil de recherche et de communication, quelles sont leurs limites?». Depuis, plusieurs numéros de la revue ont consacré une place importante aux travaux d'agronomes mobilisant la modélisation graphique, venant ainsi compléter les réponses aux questions posées dans le numéro de 1990. Certains travaux ont par ailleurs montré une évolution dans les pratiques de modélisation graphique, confirmant la richesse et la souplesse de l'outil ${ }^{1}$.

Qu'en est-il 27 ans plus tard ? La modélisation graphique a-t-elle marqué l'essai et est-elle largement mobilisée dans les travaux d'agronomie et d'aménagement des territoires? Dans quels contextes scientifiques, nouvelles questions ou nouvelles pratiques de recherche, cet outil est-il utile et utilisé, quelles sont les démarches dans lesquelles il s'insère?

3 Aujourd'hui, les problématiques saisies par les disciplines de l'agronomie et de l'aménagement des territoires sont à mettre en lien avec des enjeux globaux (alimentation, environnement, urbanisation) qui peuvent trouver leur réponse et/ou des échos à l'échelle des territoires. Plusieurs thématiques sont ainsi apparues centrales dans 
les travaux des chercheurs : rôle de l'agriculture dans la mise en place des trames vertes et bleues et plus généralement enjeux agri-environnementaux territorialisés, relations élevage et territoire (dynamique des bassins laitiers, par exemple), gouvernance alimentaire territoriale, évolution des pratiques agricoles en périurbain, modèles de développement rural-urbain.

En quoi les méthodes de la géographie (dynamiques spatio-temporelles, articulation d'échelles) trouvent-elles une place de choix pour aborder ces nouvelles problématiques, notamment par rapport à la complexité des objets analysés, du local au global ? En quoi les chorèmes peuvent-ils aider ces analyses ? Comment les chercheurs se saisissent-ils de cet outil pour aborder les problématiques majeures de l'agronomie et de l'aménagement des territoires? Face aux nouveaux objets complexes, dont les éléments constitutifs sont multi-scalaires, dans quelle mesure l'outil aide - ou limite - la prise en compte du changement d'échelle, l'intégration de différentes dimensions, l'articulation de dynamiques territoriales?

5 Par ailleurs, les pratiques de recherche ont évolué, avec la généralisation des approches systémiques, l'accès à de riches bases de données facilitant la prise en compte du changement d'échelle ou encore la multiplication des démarches participatives, impliquant plus fortement qu'avant les acteurs - des territoires notamment - à différents stades de la recherche et dans des objectifs variés (diagnostic, prospective, etc.). Comment les chorèmes et la modélisation graphique peuvent-ils appuyer nos pratiques de recherche participative? Face à l'évolution des pratiques de recherche et en particulier à la multiplication des projets participatifs, quels sont les apports et les limites de l'outil pour travailler avec les acteurs? En retour, dans quelle mesure ces nouvelles pratiques de recherche modifient la modélisation graphique ? À quels autres outils et démarches peut être associée la modélisation graphique et sous quelles formes peut se faire cette association?

6 Pour instruire ces questions et poursuivre la réflexion, ce dossier thématique propose quatre contributions, qui confirment la capacité de la modélisation graphique à être mobilisée dans des travaux à l'interface de l'agronomie et des territoires, dans des contextes variés.

\section{Les enjeux d'environnement, de production agricole et d'ancrage territorial des systèmes alimentaires}

Les quatre articles portent sur des territoires ruraux français (Livradois Forez, PoitouCharentes), mais également sur un territoire étranger (Costa Rica), et à des degrés de ruralité différents (un cas porte sur la périphérie de la ville de Millau). Différentes dynamiques rurales sont alors prises en compte et les travaux présentés dans ce dossier thématique sont représentatifs des problématiques scientifiques saisies par les disciplines de l'agronomie, de la géographie et de l'aménagement du territoire en lien avec les enjeux globaux.

8 La question de l'évolution de la production agricole, en particulier la production laitière, est abordée dans le texte de Marie Houdart (https://journals.openedition.org/ mappemonde/423) concernant la dynamique d'un bassin laitier dans le Livradois Forez. Cette réflexion s'inscrit plus généralement dans le courant des travaux sur les relations entre élevage laitier et territoire (Napoléone et al., 2015). La modélisation graphique aide 
ici à faire émerger les dynamiques à l'œuvre dans l'hybridation et la coexistence des systèmes agri-alimentaires à l'échelle du bassin laitier.

9 L'évolution des systèmes alimentaires dans les territoires est abordée sous un autre angle dans le cas des travaux d'Adrien Baysse-Lainé (https://journals.openedition.org/ mappemonde/576). La réflexion est plus intégratrice de l'ensemble des systèmes de production pouvant poser les bases du système alimentaire d'un territoire (dépassant le cas de la seule production de lait), mais constitue ce que l'auteur appelle « une approche restreinte à l'alimentation locale et adaptée à des villes de taille [...] réduite ». L'auteur propose de modéliser graphiquement la morphologie des espaces agricoles qui «nourrissent» les villes par l'intermédiaire de circuits locaux. Ces travaux s'inscrivent dans un ensemble de réflexion sur l'évolution des systèmes alimentaires, face aux enjeux sociaux et environnementaux qu'ils sous-tendent (Lardon et Loudiyi, 2014).

10 L'enjeu environnemental est plus frontalement abordé dans les articles de Muriel Bonin, Jean-François Le Coq et Fernando Saenz Segura (https://journals.openedition.org/ mappemonde/293) d'une part, et de Cécile Cot, Alain de Nayer et Guillaume Delattre ( https://journals.openedition.org/mappemonde/606) d'autre part. Dans le premier cas, l'enjeu environnemental se matérialise par des problématiques de régénération forestière en lien avec le pâturage et les auteurs s'intéressent plus généralement à la promotion des services écosystémiques en agriculture. Ils proposent des modèles spatiaux d'exploitations d'élevage $d u$ programme de reconnaissance des bénéfices environnementaux au Costa Rica. Dans le second cas, l'outil graphique est utilisé pour permettre, dans une démarche participative et multi-thématique, de faire émerger des problématiques environnementales dans les documents de planification en PoitouCharentes.

\section{Changement d'échelle, intégration de différentes dimensions et articulation de dynamiques territoriales}

11 Les thématiques abordées dans ces quatre articles ne sont pas spécifiques de l'usage de la modélisation graphique, mais bien représentatives des enjeux globaux saisis par les chercheurs de l'agronomie, de la géographie et de l'aménagement du territoire. Aborder ces thématiques en faisant usage de la modélisation graphique semble permettre d'envisager ces enjeux globaux de manière systémique. En d'autres termes, les travaux présentés dans ce dossier thématique confirment la capacité intégratrice et de prise en compte de différentes échelles spatiales et temporelles de l'outil chorématique.

12 Ainsi, dans le cas de Bonin et al., la question environnementale posée à l'échelle d'un territoire caractéristique des tensions entre pâturage et forêt est abordée à l'échelle de l'exploitation agricole. Dans le cas de la modélisation de la dynamique d'un bassin laitier, par Marie Houdart, il y a prise en compte conjointe de l'évolution des systèmes d'élevage, de celle de la filière laitière et de celle du territoire. La démarche dans laquelle s'inscrit l'outil chorématique permet alors d'intégrer des objets spatiaux multiples, aux rythmes de variation différents. Pour Cot et al., la modélisation graphique aide à rendre compte de la complexité du territoire dont les acteurs réalisent un diagnostic partagé, ses différentes dimensions et les unités spatiales qui le constituent. Quant à Baysse-Lainé, s'intéressant à l'empreinte spatiale de l'approvisionnement alimentaire local urbain, il articule dans son modèle graphique, la ville centrale, les terres agricoles des exploitations 
en circuits courts et les aires d'approvisionnement des intermédiaires de filières longues locales.

\section{Les différents usages de la modélisation graphique} œuvre de politiques publiques. Elle sert à l'élaboration de modèles théoriques pour interroger les dynamiques à l'œuvre et aider la compréhension des processus. La mise en regard de ces deux travaux donne à voir les deux archétypes de démarches de modélisation permettant d'aboutir à ce type de modèle théorique (Durand-Dastès, 1992), l'un de dynamique de bassin laitier, l'autre d'approvisionnement alimentaire local urbain. Dans le premier cas, le modèle est le résultat d'une démarche empirique: un cas particulier, le bassin laitier du Livradois Forez, sert de base de données pour construire trois modèles de dynamique de bassin laitier, qui pourront être mis à l'épreuve dans des contextes géographiques différenciés. Dans le second cas, le modèle est construit sur la base de données théoriques, définissant les secteurs d'approvisionnement en circuits courts et circuits longs d'une ville. L'application du modèle à la ville de Millau révèle un cinquième type d'approvisionnement des circuits longs.

\section{Les concepts matérialisés par la modélisation graphique}

Enfin, la richesse de la modélisation graphique, telle que présentée dans les travaux de ce dossier thématique, nous semble tenir pour beaucoup dans sa capacité à réinterroger les concepts clefs couramment mobilisés pour saisir les enjeux globaux auxquels agronomes et géographes s'intéressent (Prévost, 2005). La démarche même de formalisation graphique, en particulier dans des visées théoriques, amène le chercheur à interroger les limites de son objet, et à rentrer dans le détail de la matérialisation du concept. 
18 Ainsi, l'étude de Baysse-Lainé confirme la fluidité des échelles et des définitions du « local», qui apparaît comme une catégorie à la fois continue et discontinue, graduelle et territoriale. Dans le cas de Houdart, les notions de coexistence et d'hybridation de modèles sont en partie instruites et trouvent, dans le cas du Livradois-Forez, une application, une mise en « image ", en visibilité et en spatialisation. Dans l'étude de Bonin et al., la critique du modèle d'intensification de l'élevage se base sur des objets spatiaux matérialisés sur le terrain. Il en est de même dans l'étude de Cot et al., où c'est le dessin par les acteurs eux-mêmes des objets spatiaux et de leurs dynamiques qui fait émerger les enjeux du territoire.

\section{Perspectives pour un aménagement intégré des territoires}

19 Les articles proposés dans ce dossier thématique instruisent plusieurs questions cruciales sur les liens entre agriculture et territoire: celle des enjeux environnementaux et alimentaires, celle de l'articulation des échelles, celle de l'intégration territoriale. Les usages de la modélisation graphique pour répondre à ces questions sont divers, entre approches empiriques et approches théoriques, mais s'appuient tous sur la matérialisation des objets spatiaux et de leurs dynamiques.

D'autres pistes auraient pu être explorées, dans la lignée des travaux fondateurs, sur les démarches intégrées d'itinéraire cartographique (Deffontaines et al., 1994) ou d'aménagement intégré des territoires (Benoît et al., 2006). Les chorèmes y sont utilisés comme des principes organisateurs de l'espace (Lardon et Piveteau, 2005) qui révèlent les modèles sous-jacents aux organisations observées et fournissent un cadre d'analyse des transformations du territoire. Ils peuvent aussi être les marqueurs des représentations sociales des acteurs, repérées dans les cartes mentales (Dernat et al., 2016).

21 La prospective ouvre également des perspectives à l'usage des chorèmes, tant dans des approches théoriques qu'empiriques. Ainsi, Casanova-Enault et Chatel (2017) théorisent les dynamiques spatio-temporelles et font une démonstration convaincante de l'analyse des scénarios « Territoire Durable 2030 » du Ministère de l'Environnement ${ }^{2}$ (BIPE, 2012). Un ensemble de thématiques est cartographié pour rendre compte de la situation initiale en 2010. La modélisation graphique à base de combinaisons de chorèmes est présentée interactivement. Deux approches plus spécifiques de la biodiversité et de l'eau sont menées en parallèle, avec des cartes et des blocs-diagrammes paysagers. Les chorèmes servent aussi dans le cadre de démarches prospectives participatives (Lardon et al., 2016) tant comme grille de lecture des représentations spatiales produites par les acteurs que comme évaluation du processus d'apprentissage collectif par des objets spatiaux ayant du sens pour les dynamiques territoriales (Hertzog, 2016).

Modélisation graphique et agronomie peuvent encore s'hybrider, pour répondre aux défis des transitions territoriales en cours. Gageons que de nouveaux travaux viennent enrichir le panel existant dans les années futures ! 


\section{BIBLIOGRAPHIE}

BENOÎT M., DEFFONTAINES J.-P., LARDON S. (2006). Acteurs et territoires locaux. Vers une géoagronomie de l'aménagement. Éditions INRA, coll. « Savoir faire », 176 p. ISBN 978-2-7380-1225-8

BIPE (2012). Territoires durables 2030 : un exercice de prospective. Rapport d'étude, MEDDE, Commissariat général au développement durable, Mission Prospective.

BRUNET R. (1986). « La carte-modèle et les chorèmes ». M@ppemonde, n 86-4, p. 2-6. En ligne : https://www.mgm.fr/PUB/Mappemonde/M486/p2-6.pdf

CASANOVA-ENAULT L., CHATEL C. (2017). « La modélisation graphique de phénomènes émergents pour répondre aux besoins de la prospective ». M@ppemonde, n 119, 15 p. En ligne : http:// mappemonde.mgm.fr/119as4/

DEFFONTAINES J.-P., LARDON S. (éd.) (1994). Itinéraire cartographique et développement. INRA Éditions, 136 p. ISBN 978-2-7592-0435-9

DEFFONTAINES J.-P., CHEYLAN J.-P., LARDON S. (1990). « Gestion de l'espace rural, des pratiques aux modèles ». M@ppemonde, $\mathrm{n}^{\circ}$ 90-4, 48 p. En ligne : https://www.mgm.fr/PUB/Mappemonde/ Mappe490.html

DERNAT S., JOHANY F., LARDON S. (2016). "Identifying choremes in mental maps to better understand socio-spatial representations”. Cybergeo : European Journal of Geography, Systèmes, Modélisation, Géostatistiques, document 800, 7 p. En ligne : https://journals.openedition.org/cybergeo/27867? lang=en

DURAND-DASTÈs F. (1992). « Les modèles en géographie ». In BAILLY A., FERRAS R., PUMAIN D. (éd.), Encyclopédie de la géographie, Paris : Economica, p. 311-325.

HERTZOG T. (2016). Prospective participative en système irrigué. Construction de scénarios et jeux de rôles à l'office du Niger, Mali. Montpellier, France, AgroParisTech, thèse de doctorat.

LARDON S., PIVETEAU V. (2005). « Méthodologie de diagnostic pour le projet de territoire : une approche par les modèles spatiaux ». Géocarrefour, vol. 80/2, p. 75-90. En ligne : https:// journals.openedition.org/geocarrefour/980

LARDON S., LOUDIYI S. (2014). « Agriculture et alimentation urbaines : entre politiques publiques et initiatives locales ». Géocarrefour, vol. 89/1-2. En ligne : https://journals.openedition.org/ geocarrefour/9362

NAPOLÉONE M., CORNIAUX C., LECLERC B. (2015). Voies lactées. Dynamique des bassins laitiers entre globalisation et territorialisation. Avignon : Cardère éditeur, 313 p. ISBN 9782914053853 PRÉVOST P. (éd.) (2005). Agronomes et Territoires. Deuxième édition des Entretiens du Pradel. Paris : L'Harmattan, coll. « Biologie, écologie et agronomie », 512 p. ISBN 2296390447, VINCK D. (2009). « De l'objet intermédiaire à l'objet-frontière ». Revue d'anthropologie des connaissances, vol. $3, \mathrm{n}^{\circ} 1, \mathrm{p} .51-72$. 


\section{Quelques travaux montrant une évolution dans les pratiques de modélisation graphique}

BONIN M. (2001). « Nouvelles fonctions de l'agriculture et dynamiques des exploitations; une analyse chorématique dans les monts d'Ardèche ». M@ppemonde, $\mathrm{n}^{\circ}$ 62, p. 11-16. En ligne : https://www.mgm.fr/PUB/Mappemonde/M201/Bonin.pdf BONIN M., CARON P., CHEYLAN J.-P., CLOUET Y., THINON P. (2001). « Territoire, zonage et modélisation graphique : recherche-action et apprentissage ». Géocarrefour, vol. 76, $n^{\circ} 3, n^{\circ}$ spécial « Les territoires de la participation », p. 241-252.

BONIN M., THINON P., CHEYLAN J.-P., DEFFONTAINES J.-P. (2001). « La modélisation graphique : de la recherche au développement ». In MALEZIEUX É., TREBUIL G., JAEGER M., Modélisation des agroécosystèmes et aide à la décision. Montpellier : CIRAD/INRA, coll. « Repères », p. 391-412.

BRAU F. (2002). « Les chorèmes comme outil d'analyse des besoins exprimés par des gestionnaires du territoire ». M@ppemonde, n68, p. 7-10. En ligne : https://www.mgm.fr/PUB/Mappemonde/ M402/Brau.pdf CALMONT A., VASSOIGNE (de) C. (1999). « Guadeloupe, Martinique, Guyane : des espaces tropicaux entre insularité et continentalité ». M@ppemonde, n54, p. 10-14. En ligne : https://www.mgm.fr/ PUB/Mappemonde/M299/Calmont.pdf

CAPITAINE M., LARDON S., LE BER F., METZGER J.-L. (2001). « Chorèmes et graphes pour modéliser les interactions entre organisation spatiale et fonctionnement des exploitations agricoles ». In Géomatique et espace rural. Actes des journées Cassini 2001, Montpellier : CIRAD 16 p.

CAPITAINE M., BENOIT M. (2001). « Territoires des exploitations et finages : mutations lorraines » M@ppemonde, n 62, p. 6-9. En ligne : https://www.mgm.fr/PUB/Mappemonde/M201/CapitaineBenoit.pdf

CARON P. (2001). « Modélisation graphique et chorèmes : la gestion des parcours collectifs à Massaroca (Brésil du Nordeste) ». M@ppemonde, nº 62, p. 17-21. En ligne : https://www.mgm.fr/ PUB/Mappemonde/M201/Caron.pdf

CHEYLAN J.-P., DEFFONTAINES J.-P., LARDON S., THÉRY H. (1990). « Les chorèmes : un outil pour l'étude de l'activité agricole dans l'espace rural ?» M@ppemonde, n 4, p. 2-4. En ligne : https:// www.mgm.fr/PUB/Mappemonde/M490/PREFACE.pdf

COUIX G., DESSE M. (1992). « Îles tropicales et chorèmes ». M@ppemonde, $\mathrm{n}^{\circ}$ 3, p. 43-46. En ligne : https://www.mgm.fr/PUB/Mappemonde/M392/TROPIQUIL.pdf

DESSE M., COUIX G. (1991). « Aménagements et conflits d'usage des littoraux insulaires tropicaux au travers de chorèmes : l'exemple de la Guadeloupe et de la Réunion ». Actes du colloque international « Territoires et sociétés insulaires », Brest. Paris : Economica, p. 377-383.

HOUDART M. (2014). « La modélisation graphique ou chorématique ». In ÉTIENNE M. (éd.), Élevages et territoires. Concepts, méthodes, outils. Inra, FormaSciences, p. 87-96.

HOUDART M., BONIN M., LEPAGE C., FORT M., SAUDUBRAY F. (2005). « SIG, Chorèmes et Systèmes MultiAgents, Évolution d'un système rural martiniquais et pression polluante ». Revue Internationale de Géomatique, vol. 15, n 3, p. 339-356.

LARDON S. (2006). « Modélisation spatiale et approche agronomique ». In LEGAY J.-M., L'interdisciplinarité dans les sciences de la vie. Éditions Quæ, p. 85-102. 
LARDON S., BARON C., BOMMEL P., BOUSQUET F., LE PAGE C., LIFRAN R., MONESTIEZ P., REITZ P. (1998). Modéliser les configurations et les stratégies spatiales dans un système multi-agents pour la maîtrise de dynamiques d'embroussaillement. Conférence Modèles et systèmes multi-agents pour la gestion de l'environnement et des territoires, Clermont-Ferrand.

LARDON S., MAUREL P., PIVETEAU V. (2001). Représentations spatiales et développement territorial. Paris : Hermès Sciences, 438 p. ISBN 9782746202528.

LARDON S., PIVETEAU V. (2002). « Chorèmes et diagnostics de territoire : une expérience de formation ».M@ppemonde, $\mathrm{n}^{\circ}$ 68, p.1-6. En ligne : https://www.mgm.fr/PUB/Mappemonde/ M402/Lardon.pdf

MAIGROT J.-L. (2007). « Modélisation de l'évolution d'un finage, du rural au périurbain (Montagne bourguignonne)». M@ppemonde, $\mathrm{n}^{\circ}$ 85. En ligne : https://mappemonde-archive.mgm.fr/num13/ articles/art07101.html

MICHEL C., LARDON S. (2001). «L'accès aux espaces naturels : l'apport des chorèmes à la gestion des conflits d'usage ». M@ppemonde, $\mathrm{n}^{\circ}$ 62, p. 2-5. En ligne : https://www.mgm.fr/PUB/Mappemonde/ M201/Lardon-Michel.pdf

SILI M. (1999). « La pampa argentine : structures et évolution de l'espace rural ». M@ppemonde, $\mathrm{n}$ •53, p. 36-39. En ligne : https://www.mgm.fr/PUB/Mappemonde/M199/Sili.pdf

\section{NOTES}

1. Pour ne pas tous les citer ici, nous en proposons une sélection en fin de texte.

2. http://www.territoire-durable-2030.developpement-durable.gouv.fr/

\section{INDEX}

Thèmes : Les Chorèmes face à l'évolution des thématiques et pratiques de recherche en agronomie et aménagement des territoires

\section{AUTEURS}

\section{SYLVIE LARDON}

INRA/AgroParisTech, UMR Territoires

\section{MARIE HOUDART}

Irstea, UMR Territoires 